\title{
COVID-19 and the
}

Impact on Doctor

Wellbeing and

Training

A Mixed Methods

\section{Joseph Salem ${ }^{* 1}$, Lois Hawkins*1 ${ }^{1}$, Jessica Gates ${ }^{1}$, Ashwin Sundaram ${ }^{1}$, Yee-Ean Ong $^{1,2}$, Asanga Fernando ${ }^{1}$, Huon Snelgrove ${ }^{1}$, Mita Mistry ${ }^{1}$, Sahil Suleman $^{1}$, Indranil Chakravorty ${ }^{1,2}$}

${ }^{1}$ St Georges University Hospitals NHS Foundation Trust, London,

${ }^{2}$ St. George's University of London

* Joint first authors

Introduction - There is limited literature on how the COVID-19 pandemic has affected the wellbeing and training of junior doctors. The restructuring of rotas, redeployment of specialties and daily risk of COVID-19 exposure is likely to have had a significant impact on frontline doctors.

Aim - To understand the impact of COVID-19 on wellbeing and training of junior doctors at a tertiary, London Hospital.

Methods - A mixed methods study was undertaken with an initial online survey three weeks after the peak of the COVID-19 surge; followed by a series of focus groups.

Results - Of 541 junior doctors, 161 responded to the questionnaire and 10 participated in focus group sessions Over a third $(34 \% ; n=47)$ were concerned about the risk to personal health, $71 \%(n=102)$ had impaired sleep and many changed their lifestyles to adapt. Almost $40 \%$ felt the pandemic had an adverse impact on their careers, including their ability to complete training requirements, leading to an inevitable need to extend training. There was a reluctance to show or share any personal anxiety or vulnerability at work, hence participation in organised psychological support/ debrief sessions and online resources was considered unhelpful.

Conclusion - Employers need to recognise the impact of COVID-19 on the wellbeing of doctors and implement strategies to effectively support staff. The development of safe, timely and confidential psychological support strategies may be of benefit to doctors. National training leads will need to closely supervise training changes appreciating both the variation in expectations and adaption required across different specialties and grades.

Keywords junior doctors, wellbeing, COVID-19

\section{joseph.salem@nhs.net lois.hawkins@nhs.net}

Cite as; Salem, J., Hawkins, L., Gates, J., Sundaram, A., Ong, Y-E., Fernando, A., Snelgrove, H., Mistry, M., Suleman, S. \& Chakravorty, I. (2020) COVID-19 and the impact on doctor wellbeing and training- $A$ mixed methods study. The Physician vol 6; issue 3; epub 05.10.2020 DOI: $10.38192 / 1.6 .3 .2$

\begin{tabular}{ll}
\hline Submitted & 03.10 .2020 \\
Epub & 05.10 .2020
\end{tabular}

Peer reviewed by JS Bamrah, Arun K Gupta \& John Launer

Revised 22.01.2021

Creative Commons Licence 4.0 CC-BY-ND-4.0

\section{Article Information}

\section{Introduction}

The COVID-19 pandemic has had a significant impact on both the emotional wellbeing of doctors (1) and their ongoing training (2). While, the rest of the world went into lockdown, healthcare workers continued working in physically and emotionally difficult circumstances with an increasing caseload and acuity of patients coming into hospital. Junior doctors traditionally have had to endure great workplace stress (3), often do not prioritise their own health (4), feel pressured not to miss shifts because of their 
own health problems (5) and up to $80 \%$ are high risk for burnout (6). This is likely to have worsened during the recent pandemic with a recent British Medical Association (BMA) survey finding that $35 \%$ of hospital doctors considered their mental health to be worse during the period (7). Whilst there has been some work looking at medical wellbeing in China and Italy $(8,9)$ there is limited data assessing the impact on training and wellbeing of trainees within the United Kingdom (UK). Our aim was to explore the perceptions of junior doctors on the impact of COVID-19 on training and wellbeing using a mixture of qualitative and qualitative methods.

\section{Methods}

\section{Study environment}

This study was conducted at St. George's Hospital, a tertiary centre in London, which admitted 895 patients with COVID-19 with the peak admission date being the week of the $25 / 3 / 2020$. As a consequence, junior doctor rotas were adapted to increase medical staffing in the frontline. Existing rotations were paused and acute medical, non-acute medical (medical specialties that are not included on the acute medical rota) and surgical doctors were redeployed either to intensive care units or to 'megateams' for acute medicine. The megateams were implemented from 27/03/2020 and included a selection of doctors from a variety of specialties and grades looking after COVID-19 and non-COVID-19 medical in-patients.

This study was conducted after the lockdown was announced on the 23/3/2020 and after the peak of admissions had passed at St. George's Hospital but before the initial lockdown was eased (4/07/2020 (10)) or recommendations for the use of personal protective equipment (PPE) in certain areas of public life (15/06/2020 (11)).

\section{Study design \& Methods}

This was a mixed method study using a questionnaire and focus groups.

Phase I - An anonymised questionnaire was administered to junior doctors by email through the Postgraduate Medical Education team as well as through and professional social media groups during the peak of the COVID-19 surge in the UK. Responses were collected using the SurveyMonkey ${ }^{\circledast}$ tool (12) regarding 3 domains: redeployment, wellbeing and training and were analysed using Microsoft Excel (13) Phase II - Junior doctors were invited via email and professional social media groups to attend semi structured focus groups. The focus groups were conducted with written consent, face to face or online using a video platform (Microsoft Teams(14)).
Questions are outlined in Appendix 1. Institutional ethics approval was obtained. Qualitative data was gathered, transcribed and analysed using thematic analysis. This project was covered by the St Georges University Research \& Ethics Committee institutional approval number: SGREC18.0008.

\section{Outcomes}

(i) Health \& Wellbeing - sleep, intensity of work, lifestyle changes, work-life balance, support received, risk of COVID-19

(ii) Redeployment - multidisciplinary skill mix, patient care

(iii) Training and clinical competency

\section{Results}

A total of 161 responses from a potential cohort of 541 junior doctors working at the hospital was collected. The respondents were $58 \%$ female, $65 \%$ white and $30 \%$ had spent more than 4 weeks on COVID-19 wards. There was a mixture of doctor grades as seen in Table 1.

\section{Health \& Wellbeing}

Sleep - The majority $(71 \%, n=102)$ suffered sleep disturbance with $67 \%(n=64)$ of these doctors reporting this disturbance staying the same or worsening throughout the pandemic period.

Sources of support - Eight-two percent of doctors ( $n=112$ ), felt supported clinically with $(39 \%, n=53)$ feeling more supported than before the pandemic. Doctors commented on the benefits such as "reduced sense of hierarchy", "increased staffing levels", "greater levels of senior support" and feeling "less likely to burn out or need time off". Most respondents sought support from friends and family $(75 \%, n=100)$, followed by informal peer support $(56 \%, n=76)$ or from senior doctors $(40 \%, n=55)$. These can be seen in Figure 1.

Work-life balance - Half of all doctors $(51 \%, n=65)$ felt they were less busy than usual, $35 \%$ felt work-life balance had deteriorated, while $29 \%(n=38)$ felt work life balance had improved. Medical trainees reported the biggest improvement in work life balance $(46 \%, n=13)$. A large proportion of comments related to challenges with frequent changes to rostered duties and rates of pay as well as "lack of communication" between departments.

Risk of COVID-19 - Forty two percent of respondents reported having symptoms compatible with COVID-19, although only $12 / 17$ who had been swabbed had laboratory confirmation. Twenty-four percent $(n=33)$ were obliged to self-isolate due to household members having symptoms. Over one third of doctors reported a high level of concern (defined as $\geq 7 / 10$ on 
a Likert scale) regarding the risk of exposure to COVID-19 patients, this increased after deployment. Black, Asian and Minority Ethnic (BAME) respondents reported higher levels of concern for their health, both before and after starting on COVID wards. The most common adjustment to the daily routine was changing clothes when arriving home, increasing

\begin{tabular}{|l|r|}
\hline $\begin{array}{l}\text { Table 1: Demographics of the } \\
\text { participants }\end{array}$ & Total \\
\hline Characteristics & N (\%) \\
& \\
\hline Gender & $65(40.4)$ \\
Male & $94(58.4)$ \\
Female & $2(1.3)$ \\
Did not wish to state & \\
\hline Ethnicity & $104(64.5)$ \\
White & $38(23.6)$ \\
Asian & $3(1.8)$ \\
Black & $12(7.4)$ \\
Other & $4(2.5)$ \\
Did not wish to state & \\
\hline Amount of time spent on & $45(30)$ \\
COVID-19 or suspected wards & $83(51.5)$ \\
>4 weeks & $33(20.5)$ \\
<4 weeks & \\
Not applicable & \\
\hline Training year & $24(14.9)$ \\
F1 & $22(13.7)$ \\
F2 & $22(13.7)$ \\
Clinical fellow/locum & $17(10.6)$ \\
Core medical trainee & $4(2.5)$ \\
Core surgical trainee & $5(3.1)$ \\
Other core trainee & $12(7.5)$ \\
Acute medical registrar & $10(6.9)$ \\
Non-acute medical Registrar & \\
Surgical Registrar & \\
Other & \\
& \\
\hline
\end{tabular}

their household cleanliness and changing their mode of transport to work. Twenty two percent $(n=31)$ of trainees changed their living arrangements, with $52 \%$ of those who made changes $(n=13)$ moving to different accommodation to protect their families. 


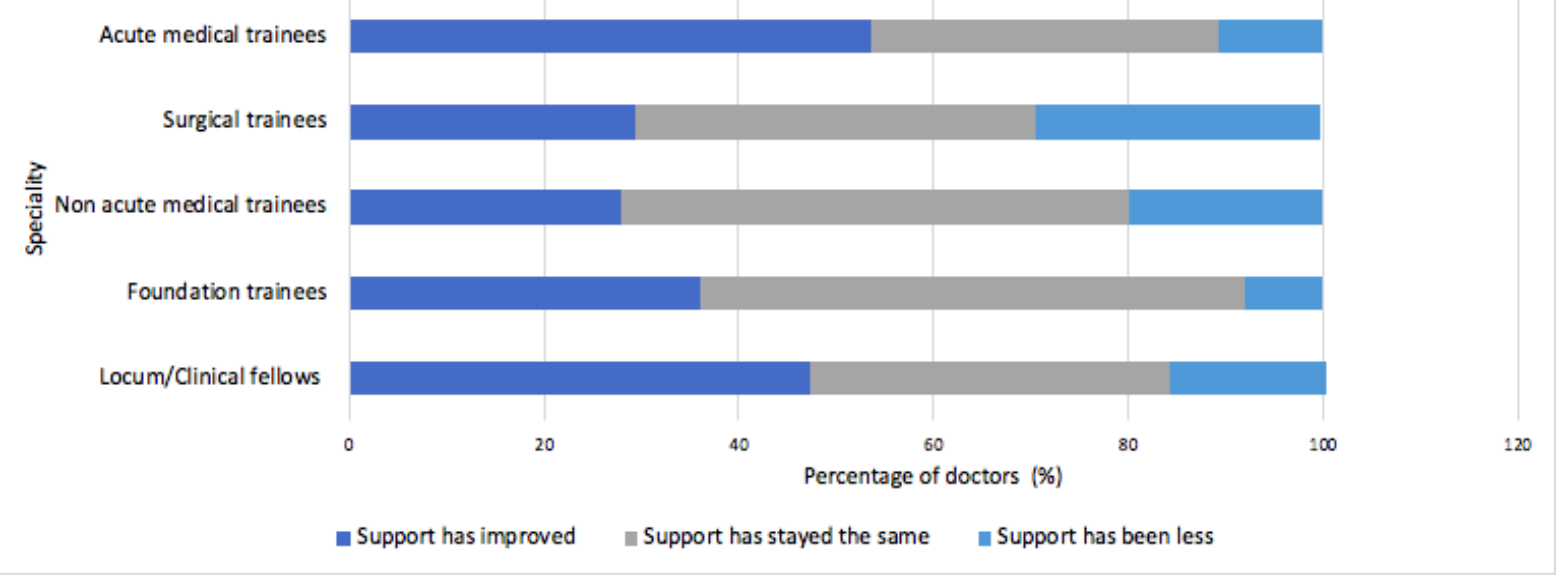

Personal Protective Equipment (PPE) - Forty three percent $(n=58)$ were not confident following Public Health England's (PHE) PPE guidance due to lack of congruence with recommendations from the World Health Organisation (WHO), but $69 \%$ felt they had adequate access to PPE. One third of BAME doctors were less confident with PHE's guidance and 59\% reported inadequate PPE. Only $16 \%(n=22)$ of doctors used masks and $10 \%(n=14)$ used gloves outside the hospital.

\section{Redeployment \& Competency}

Twenty-six percent of doctors $(n=34)$ reported high levels of concern ( $\geq 7 / 10$ on a Likert scale) for their clinical competency in managing patients with COVID-19. Surgical trainees (38\%, $\mathrm{n}=6$ ) were the most concerned group, in comparison to foundation doctors $(26 \%, n=10)$, acute medical trainees $(26 \%$, $\mathrm{n}=7)$, locum/clinical fellows $(21 \%, \mathrm{n}=4)$ and non-acute medical trainees $(18 \%, n=4)$. A third of acute medical trainees (33\%, $\mathrm{n}=9$ ) felt having doctors from a range of different medical and Figure 2: Doctor's concerns regarding the effect of COVID-19 on training

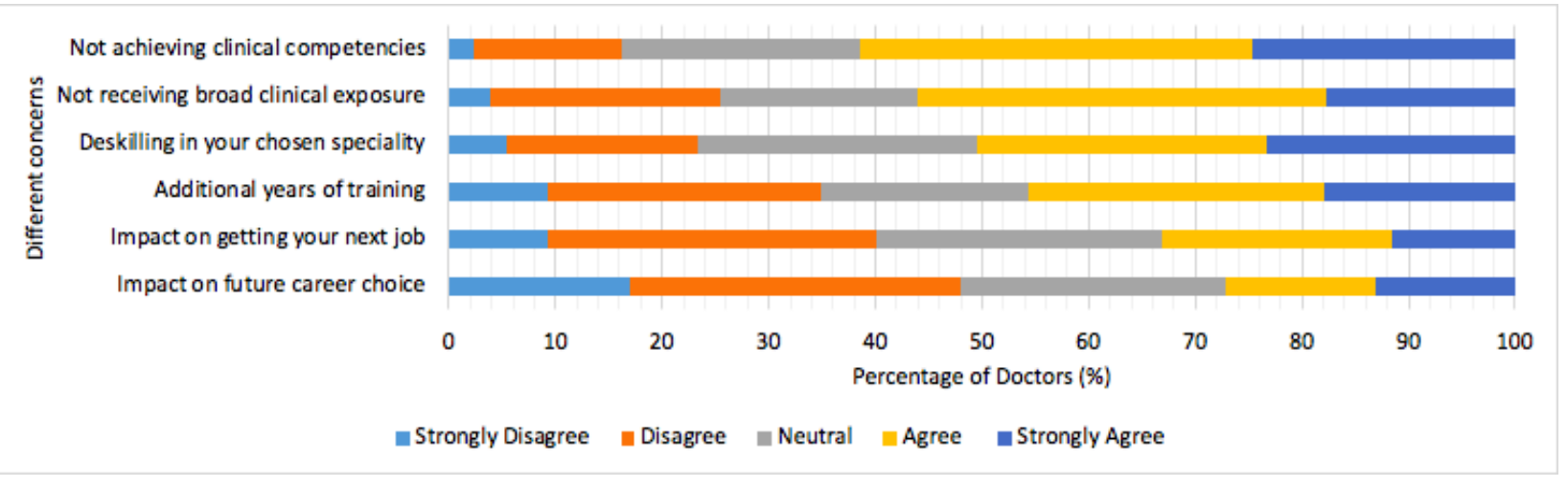

\section{Focus Groups}

Three focus group sessions were arranged with 10 participants including foundation, core and higher speciality surgical specialty backgrounds had a positive impact on care compared to $0 \%(n=0)$ of surgeons, $12.5 \%(n=3)$ of non-acute specialities and $18 \%(n=7)$ of foundation doctors.

\section{Impact on Training}

Overall, $40 \%(n=52)$ felt that the COVID-19 pandemic is going to have a lasting effect on their training, most apparent in surgical (47\%; $n=7$ ) and non-acute medical speciality trainees $(50 \% ; n=12)$. The most common concerns were failure to achieve clinical competencies, not receiving a broad clinical exposure and deskilling in their chosen speciality. While the majority of doctors $(58 \%, n=75)$ felt their training should not be extended, $44 \%(n=7)$ of surgeons, and $50 \%(n=12)$ of nonacute specialities preferred their training to be extended compared to acute medical trainees $(11 \%, n=3)$ and foundation doctors $(11 \%, n=4)$, (see Figure 2 ). The majority of doctors abandoned recording training events in their eportfolios during the pandemic. 
phase of the COVID-19 surge. The analysis of transcripts allowed us to categorise emerging themes into two broad descriptive categories divided into several subsections that can be seen in Table 2.

Table 2: Categories of themes identified from focus groups

\begin{tabular}{|l|l|l|l|}
\hline Health and wellbeing & Professional practice \\
\hline Personal Wellbeing & Leaning to cope & Redeployment & Learning \\
\hline Daily life & Support & Competency & Training \\
\hline Mental health & Coping & Interdisciplinary working & Leadership \\
\hline Physical health & Targeted Resources & Communication & Return to work \\
\hline
\end{tabular}

\section{Theme 1- Personal Wellbeing}

Mental health - Participants described anxiety about passing COVID-19 on to their family members, rather than anxiety about contracting the virus themselves. The impact of having to self-isolate from their families and the angst from the frequently changing advice on social distancing or PPE added further worry and frustration. They recognised the challenge of having to care for patients in great distress (due to hypoxia or delirium), and the difficulty to empathise or physically connect with them to offer comfort, including having to decline access to their next-of-kin. Some experienced making resuscitation or end-of-life decisions with limited knowledge of pre-existing conditions as violations of their core beliefs. Furthermore, this mental distress could be amplified when having to certify a number of deaths during a single shift, or not having time to de-brief with colleagues. Taking their distress back home with them at the end of the shift made it hard to feel 'off duty'. The isolation from friends and family outside of the work environment also took its toll.

Physical Health - Participants described being exhausted at end of long shifts, sometimes finding that irrelevant events tended to bring out emotional upheavals. Some found sleep being interrupted, often waking in the night in 'a cold sweat' and wondering whether they had acquired the virus. Some who had the disease described the profound fatigue which lasted weeks and the isolation that accompanied the mandatory quarantine. Others expressed anger at the difficulty getting tested once symptoms did develop. Common lifestyle reports included lack of exercise due to lack of leisure time and facilities; the involuntary consumption of high calorific food, often deemed 'unhealthy', and the impact on weight and body image.

Support - Participants did not believe that sharing one's emotional vulnerabilities was helpful for team morale and did not believe that the team would perform better if such emotions were shared. Participants were broadly unaware of the range of structured support available and many had limited expectations from the organisation. Some appreciated the existence of a 'wellbeing hub' where one could relax with free access to coffee or refreshments. This resource was appreciated due to the ability to 'get away from it all' for a period of quiet reflection or for interaction with peers. Online resources were broadly ignored or not considered helpful. For some, psychologically-informed reflective or debrief sessions were missed because they did not happen at opportune times or were perceived not to happen at all. The sessions offered by the organisation were not popular among the participants mainly because they felt they were 'mandated' and secondly because of the 'anxiety associated with exposure of one's inner self or psychological vulnerability' in front of colleagues.

Personal strategies included sleep, exercise yoga or meditation. There was little time to seek comfort from the arts, music or television although having other lifestyle dimensions - partners, faith groups, gardening - did provide some solace. There was limited appetite for participating in targeted resources such as wellness webinars, web-based tools, podcasts or mindfulness apps.

\section{Theme 2 - Redeployment \& Competency}

Clinical challenges included anxieties about ability to safely upskill to the clinical procedures and rapid decision-making that would inevitably be necessary, especially among participants less experienced in intensive care or acute medical areas. Most participants agreed that the redeployment initiative by the organisation was effective in offering a manageable, albeit intensive workload. The additional doctors deployed on nights, weekends and the 'mega firms' offered a structure that was clearly effective and appreciated. There were emotional challenges experienced having to work with unfamiliar members of the redeployed teams, being unfamiliar with the skill-set of the teams and the level of responsibility that could be delegated safely. Communicating effectively through masks and visors was felt to be a problem both with patients and team members.

\section{Theme 3- Training}

Training - Participants were anxious about missing their clinical competencies, their training requirements and the inadvertent need for extension of their training. There was concern regarding the need to catch up on missed examination, clinical competency and procedural requirements as well as the delay in achieving completion of training. 
Return to work - There was mixed feelings about the justification of the delayed institution of measures such as mandatory requirement for wearing masks in public places and concern returning to over-crowded public transport. There were challenges of not knowing shift patterns or clinical areas of allocation which were altered frequently, as the organisation coped with a rising surge of patients. Participants who had recovered from COVID-19 infection felt relieved and safer to continue working although others felt frustration at the changing testing regimes.

\section{Discussion}

Our study explored the impact of the COVID-19 pandemic on the wellbeing and training of junior doctors, and has highlighted the physical stresses and the psychological pressure that doctors experienced, but also their adaptability, their coping strategies and their resilience. There were concerns regarding the personal risks (15), risks to vulnerable family (16), conflicting guidance on PPE (17), challenging ethical dilemmas in decision-making and witnessing multiple deaths. There were challenges from changing rotas during redeployment, anxieties about missed training opportunities particularly among procedure-based specialties such as surgical trainees as well as the abandonment of e-portfolios. The daily contact with COVID-19 led to significant lifestyle changes for many doctors including moving out of their homes, avoiding public transport and restricting contact with family members.

Current evidence has shown high anxiety and depression levels amongst frontline workers in China (18) which highlights the importance of an available and accessible support system within the work environment (19). However despite this, only $7 \%$ of doctors in the hospital accessed organisational support resources. The reason for this lack of uptake is likely to be multifactorial. Support sessions have been associated with stigmatisation amongst health professionals, which is likely to be a barrier to doctors attending them (20). Doctors reflected that they were reluctant to reveal their wellbeing concerns at work, and there appeared to be greater psychological safety in 'opening up' to peers, rather than relying on support through psychologically-informed reflective sessions.

The professional challenges emerging from interviews related to the difficulty at times to reconcile the discrepancy between the doctor's core belief - ability to help, treat or cure - and the perniciously realistic threats posed by the virus. Participant descriptions of intrusive thoughts, sleeplessness or sometimes guilt emerged as familiar reactions to stress, but also inappropriate 'coping' strategies following the exceptional challenges to their physical and psychological security.

It is known that leaders have a significant impact on team safety and a protective role regarding junior doctor wellbeing (21). Our organisation has developed guiding principles and a framework to support clinical and educational supervisors to proactively engage with a process of actively monitoring junior doctor wellbeing and offering a 'check-in' interview and associated action plan informed by evidence-based practice (22).

Training is undoubtedly going to be affected by this pandemic, and we will need to adapt to deliver training in new and different ways in the aftermath of COVID-19. Across all specialties, trainees had concerns regarding non-completion of core competencies along with reduced clinical exposure. The majority of surgeons believe that training should be extended, which may be related to the hiatus in elective procedures, a greater proportion of consultant-led surgeries and less in-theatre training for surgeons. Medical specialties, however, do not feel an extension is necessary, probably because patients with COVID-19 were medical patients and thus these experiences contributed to their training.

There is concern that the COVID-19 pandemic will have a longlasting effect on training, which is in keeping with other data where doctors reported similar concerns (23). This has been apparent in doctors who have been applying for specialty, core and general practice training jobs during the pandemic. These individuals experienced significant disruption during the recruitment process including the cancelling of interviews and adaptations to point-weighting putting some trainees at a disadvantage without any prior warning. Health Education England (HEE) will need to closely supervise training changes appreciating both the variation in expectations and adaption required across different specialties and grades (24).

The main limitations of this survey are the reduced response rate and that the views of doctors were only sampled from a single hospital in London. Despite many similar changes occurring nationwide the small sample size does limit the ability to make generalisations about the results. The focus groups were limited to a small number of respondents, most of whom were female. Nevertheless, this data adds to a paucity of literature on how doctors felt in the epicentre of the pandemic and will help provide important information regarding planning for potential second waves of COVID-19 or other pandemics.

\section{CONCLUSION}

With ever-changing government and hospital advice, the views of junior doctors and how this pandemic has affected their wellbeing and training is likely to evolve. HEE (2019) have recently stated 'we must improve the way in which we look after ourselves and our colleagues so they are better placed to meet the needs of patients'(25). The COVID-19 pandemic may be an opportunity to re-evaluate how we better manage the pressure and high demands on staff in the short and longer term. Hospital trusts need to build effective tools that can enable junior doctors to report concerns, communicate changes affecting normal work routines early 
and effectively and implement adequate feedback to ensure trainees feel supported in all aspects of their training.

\section{References}

1. McAlonan, G. Lee, A. Cheung, V. Tsang, K. Sham, P. Chua, S. Wong, J. Immediate and Sustained Psychological Impact of an Emerging Infectious Disease Outbreak on Health Care Workers. The CanJ Psychiat. 2007;52(4):241-247.

2. Willan, J. King, A. Jeffrey, K. Bienze, N. Challenges for NHS hospitals during covid-19 epidemic. BMJ. 2020;368:m1117

3. Shanfelt, T. Bradley, K, Wipf, J. Back A. Burnout and selfreported patient care in an internal medicine residency program. Ann Intern Med. 2002; 136(5):358-67.

4. Markwell, A. Wainer, Z. The health and wellbeing of junior doctors: insights from a national survey. Med J Australia. 2009; 191(8): 441- 4 .

5. Shadbolt, N. Attitudes to healthcare and self-care among junior medical officers: a preliminary report. Med J Australia. 2002; 177 (S1).

6. British Medical Association. Caring for mental health of the medical workforce. Available from URL:

https://www.bma.org.uk/media/1365/bma-caring-for-themental-health-survey-oct-2019.pdf [Accessed August 2020].

7. British Medical Association. COVID-19: analysing the impact of coronavirus on doctors. Available from URL:

https://www.bma.org.uk/advice-and-support/COVID-19/what ${ }^{-}$ the-bma-is-doing/COVID-19-analysing-the-impact-ofcoronavirus-on-doctors [Accessed August 2020]

8. Cao, J. Wei, J. Zhu, H. et al. A Study of Basic Needs and Psychological Wellbeing of Medical Workers in the Fever Clin. of a Tertiary General Hospital in Beijing during the COVID-19 Outbreak. Psychother Psychosom. 2020;89(4):252-254.

9. Amerio,A. Bianchi, D, Santi, F. et al. COVID-19 pandemic impact on mental health: a web-based cross-sectional survey on a sample of Italian general practitioners. Acta Biomed. 2020;91(2) 83-88.

10. Department of Health and Social Care. Face masks and coverings to be worn by all NHS hospital staff and visitors. Available from URL:

https://www.gov.uk/government/news/face-masks-andcoverings-to-be-worn-by-all-nhs-hospital-staff-and-visitors [Accessed July 2020].

11. Department of Health and Social Care. PM announces easing of lockdown restrictions. Available from URL: https://www.gov.uk/government/pm-announces-easing-of lockdown-restrictions-23-june-2020. [Accessed July 2020]

12. SurveyMonkey. The World's Most Popular Free Online Survey Tool. Available from URL: https://www.surveymonkey.com/ [Accessed July 2020].

13. Microsoft Corporation. Microsoft Excel. Available from URL: https://office.microsoft.com/excel [Accessed July 2020]

14. Microsoft Corporation. Microsoft Teams. Available from URL: https://www.microsoft.com/en-gb/microsoft-365/microsoftteams/group-chat-software [Accessed July 2020]

15. Wang, W. Tang, J. Wei, F. Updated understanding of the outbreak of 2019 novel coronavirus (2019-nCoV) in Wuhan, China. J. Med. Virol. 2020;92(4):441-7.

16. Brooks, S. Webster, R. Smith, L. Woodland, L. et al. The psychological impact of quarantine and how to reduce it: rapid review of the evidence. Lancet. 2020; 395: 912-20.
17. Herron J, Hay-David A, Gilliam A, Brennan P. Personal protective equipment and COVID 19-a risk to healthcare staff?. Brit J Oral Max Surg. 2020;58(5):500-2.

18. Lai, J. Ma, S. Wang, Y. Factors associated with mental health outcomes among health care workers exposed to coronavirus disease 2019. JAMA netw open. 2020;3(3):e203976.

19. Wu, P. Styra, R. Gold, W. Mitigating the psychological effects of COVID-19 on health care workers. Can Med Assoc J. 2020;192(17):E459-60.

20. Cohen, D. Winstanley, S. Greene, G. Understanding doctors' attitudes towards self-disclosure of mental ill health. Occup Med. 2016;66(5):383-389.

21. Wu, A. Connors, C. Everly, G. COVID-19: peer support and crisis communication strategies to promote institutional resilience. Ann Intern Med. 2020;172(12):822-823.

22. Tracy, D. Tarn, M. Eldridge, $R$.et al. What should be done to support the mental health of healthcare staff treating COVID19 patients? BrJ Psych. 2020;1-3.doi:10.1192/bjp.2020.109

23. Federani, R. Monks, M. Peprah D .et al. Improving wellbeing among UK doctors redeployed during the COVID-19 pandemic. Future Healthc J. 2020; 7.3 DOI:10.7861/fhj.2020-0111

24. Daodu, O. Panda, N. Lopushinsky, S. Varghese, T. Brindle, M. COVID-19-considerations and implications for surgical learners. Ann Surg. 2020;1;272(1):e22-3.

25. Health Education England. NHS Staff and Learners' Mental Wellbeing Commission. Available from URL:

\section{https://www.hee.nhs.uk/sites/default/files/documents/NHS\%20\%2} 8 HEE $\% 29 \% 20$ -

20Mental\%20Wellbeing\%20Commission\%20Report\%20\%28Summa ry\%29.pdf [Accessed July 2020]

\section{Author contribution}

JS \& LH: Project lead, Project conception, Study design, Data collection, Data analysis and interpretation, writing of manuscript, reviewing manuscript, editing of manuscript

AS: Administrative support, Reviewing manuscript

JG \& YO: Conception of project, Study design, Reviewing manuscript, Supervision of manuscript

AF \& HS \& MM: Data collection, Data analysis and interpretation, Editing of manuscript, Administrative support

SS: Study design, Data collection, Editing of manuscript, Reviewing manuscript, Supervision of manuscript

IC: Study design, Data collection, Data analysis and interpretation, Editing of manuscript, Reviewing manuscript, Supervision of manuscript

\section{Funding}

No funding was required for this project

\section{Conflict of Interests statement}

The authors have no conflict of interest to declare. Indranil Chakravorty (IC), is an honorary members of BAPIO Institute for Health Research, Bedford, UK. BAPIO is a national professional body of multi-professional health care workers in the UK since 1996 (https://www.bapio.co.uk/).

\section{Acknowledgements}

We would like to thank the Postgraduate Medical Education centre at St. George's Hospital NHS Foundation Trust for their help with the administration of this project. 
